\section{CD24FC AMELIORATES IMMUNE-RELATED ADVERSE EVENTS WHILE PRESERVING ANTI-TUMOR THERAPEUTIC EFFECT}

${ }^{1}$ Mingyue Liu*, 'Xu Wang, 'Peng Zhang, 'Juanjuan Su, 'Xuexiang Du, 'Yan Zhang, ${ }^{2}$ Yang Liu, ${ }^{2}$ Pan Zheng. ${ }^{1} I H V / U M B$, Baltimore, MD, United States; ${ }^{2}$ OncoC4, Inc., Rockville, MD, United States

Background Combination therapy with anti-CTLA-4 and antiPD-1 mAbs has emerged as the most potent and durable cancer immunotherapy, yet it is associated with frequent and severe immune-related adverse events (irAEs). ${ }^{1}{ }^{2}$ A largely unmet medical need is to reduce irAEs. The CD24-Siglec 10/ $G$ interaction is an emerging immune checkpoint that regulates inflammation caused by danger-associated molecular patterns (DAMPs). ${ }^{3-5}$ It is of great interest to investigate whether CD24Fc can ameliorate severe irAEs, the hallmark of which is a severe inflammatory state in multiple organs.

Methods We used a human CTLA-4 knock-in (Ctla4h/h) mice model that fully recapitulates human irAE in response to antiPD-1 and anti-CTLA-4 antibodies to test if CD24Fc have therapeutic effect for irAE. We treated Ctla4h/h mice with Ipilimumab and anti-PD-1 $\mathrm{Ab}$ in conjunction with hIgFc or $\mathrm{CD} 24 \mathrm{Fc}$ on day $10,13,16$ and 19 after birth. The body weight was monitored over time, hematologic and histopathologic alterations were evaluated at 6 weeks of age. To evaluate the therapeutic effect of $\mathrm{CD} 24 \mathrm{Fc}$ on ICIs induced tissue destruction, we performed histological analysis of internal organs and glands. Major organs were collected about 1 month after first treatment and fixed in 10\% formalin, sectioned and stained with hematoxylin and eosin (H\&E), and scored double blindly. To test whether CD24Fc immune modulation may interfere with the anti-tumor efficacy of the checkpoint inhibitors, we inoculated MC38 and B16-F10 tumor cells on $\mathrm{Ctla} 4 \mathrm{~h} / \mathrm{h}$ mice, then treated with combination of Ipilimumab and anti-PD-1 Ab together with hIgFc or CD24Fc and monitored tumor growth.

Results We found that anti-CTLA-4 and anti-PD-1 therapy could induce growth retardation, anemia and severe inflammation in all organs examined. All of these adverse events were ameliorated by CD24Fc treatment. Moreover, in both tumor models tested CD24Fc modestly enhanced immunotherapeutic effect of anti-PD-1 and anti-CTLA-4 antibodies. CD24Fc treatment showed no effect on CD4+, CD8 $+\mathrm{T}$ cell or tumor associated macrophage (TAM) density intratumor. However, we observed significantly decreased Treg among CD4+T cells after $\mathrm{CD} 24 \mathrm{Fc}$ treatment. CD24Fc treatment also decreased the TIM-3 + PD-1+ CD4+ and CD8 + T cells. These data suggest $\mathrm{CD} 24 \mathrm{Fc}$ has the potential to optimize tumor microenvironment and augment antitumor immunity.

Conclusions Our data demonstrate that CD24Fc treatment ameliorates irAEs in multiple organs induced by combination of anti-CTLA-4 and anti-PD-1 Abs while modestly enhancing its anti-tumor activity, potentially by reducing the intratumor regulatory $\mathrm{T}$ cells and reverse exhaustion of tumor-infiltrating T cells.

\section{REFERENCES}

1. Wolchok JD, et al. Overall survival with combined nivolumab and ipilimumab in advanced melanoma. N Engl J Med 2017;377:1345-1356.

2. Larkin J, et al. Five-year survival with combined nivolumab and ipilimumab in advanced melanoma. N Engl J Med 2019;381:1535-1546.

3. Chen GY, Tang J, Zheng P, Liu Y. CD24 and Siglec-10 selectively repress tissue damage-induced immune responses. Science 2009;323:1722-1725.

4. Liu Y, Chen GY, Zheng P. CD24-Siglec G/10 discriminates danger- from pathogen-associated molecular patterns. Trends Immunol 2009;30:557-561.
5. Fang $X$, Zheng $P$, Tang J, Liu $Y$. CD24: from A to Z. Cell $\mathrm{Mol}$ Immunol 2010:7:100-103.

http://dx.doi.org/10.1136/jitc-2021-SITC2021.813 